\title{
Stopping power dependence of nitrogen sputtering yields in copper nitride films under swift-ion irradiation: Exciton model approach
}

\author{
N. Gordillo \\ R. Gonzalez-Arrabal \\ A. Rivera \\ F. Munnik \\ F. Agulló-López
}

\begin{abstract}
A B S T R A C T
Nitrogen sputtering yields as high as $10^{4}$ atoms/ion, are obtained by irradiating $\mathrm{N}$-rich- $\mathrm{Cu}_{3} \mathrm{~N}$ films ( $\mathrm{N}$ concentration: $33 \pm 2$ at.\%) with $\mathrm{Cu}$ ions at energies in the range $10-42 \mathrm{MeV}$. The kinetics of $\mathrm{N}$ sputtering as a function of ion fluence is determined at several energies (stopping powers) for films deposited on both, glass and silicon substrates. The kinetic curves show that the amount of nitrogen release strongly increases with rising irradiation fluence up to reaching a saturation level at a low remaining nitrogen fraction $(5-10 \%)$, in which no further nitrogen reduction is observed. The sputtering rate for nitrogen depletion is found to be independent of the substrate and to linearly increase with electronic stopping power $\left(S_{e}\right)$. A stopping power $\left(S_{t h}\right)$ threshold of $\sim 3.5 \mathrm{keV} / \mathrm{nm}$ for nitrogen depletion has been estimated from extrapolation of the data. Experimental kinetic data have been analyzed within a bulk molecular recombination model. The microscopic mechanisms of the nitrogen depletion process are discussed in terms of a non-radiative exciton decay model. In particular, the estimated threshold is related to a minimum exciton density which is required to achieve efficient sputtering rates.
\end{abstract}

\section{Introduction}

Copper nitride is a semiconducting material with an optical

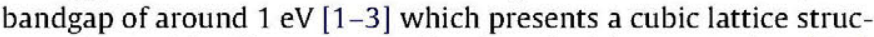
ture. $\mathrm{Cu}_{3} \mathrm{~N}$ thin films are usually deposited by sputtering $[4,5]$. Being metastable [6], copper nitride easily decomposes into metallic copper and nitrogen by different techniques such as: thermal annealing [6-9], and irradiation with electrons and laser pulses [10-13]. More recently, the effect of swift heavy ion (SHI) irradiation [14-16] in which the electronic stopping power $\left(S_{e}\right)$ is some orders of magnitude higher than the nuclear stopping power $\left(S_{n}\right)$ is being investigated. When the material decomposes, it simultaneously undergoes large changes in electrical resistivity and reflectivity which opens the possibility to use it for write-once optical data storage. Moreover, irradiation methods have been used to pattern the material producing sub-micrometric conductive dots and lines which have a signal speed higher than those usually used in integrated circuits [9]. SHI irradiation presents some clear advantages with respect to the other employed patterning techniques since energetic ions deposit their energy in the material by electronic excitation, forming structures (tracks) with nanometer lateral resolution $[17,18]$. Thus, SHI irradiation is a clean process which does not require any type of resist or cleaning procedures for patterning. Diverse phenomenological models such as: coulomb explosion [18], thermal spike [19] and excitonic [20,21] have been proposed to describe the responsible mechanism for track formation. The main difference between them is the way in which the SHI transfers its energy to the target lattice. So far, most previous works about track formation have been performed on insulating materials with large electronic bandgaps, like $\mathrm{LiNbO}_{3}$, in which a rather coherent picture is emerging $[17,22,23]$. However, for low bandgap semiconductors, like InP and InAs the situation is still quite complex and the processes of damage and defect formation are not sufficiently understood [24-27]. Some previous studies carried out on metal nitrides irradiated with SHI evidence that nitrogen sputtering is the main direct effect of irradiation [16,28-30].

The study of radiation-induced damage in metal nitrides presents the advantage in the sense that the number of sputtered nitrogen atoms can be easily monitored by elastic recoil detection (ERD). Therefore, it provides a very direct and sensitive method to investigate the irradiation effect and learn about damage mechanisms.

The purpose of this work is to investigate the kinetics of nitrogen sputtering from the film as a function of ion fluence and 
stopping power for samples irradiated with $\mathrm{SHI}$. To this aim $\mathrm{N}$-rich $\mathrm{Cu}_{3} \mathrm{~N}$ thin films were irradiated at room temperature with $\mathrm{Cu}$ ions at various MeV energies to cover a broad span of electronic stopping powers. The sputtering efficiency is determined as a function of irradiation fluence and electronic stopping power. As far as we know, the latter dependence, which plays a key role in the understanding of damage mechanisms, is reported here for the first time. A stopping power threshold for nitrogen depletion is estimated to be $S_{t h} \sim 3.5 \mathrm{keV} / \mathrm{nm}$. The gathered kinetic data, discussed on the basis of the bulk molecular recombination model (from now on BMR) developed by Adel et al. [29] provide reliable information on the processes leading to bond-breaking and nitrogen liberation. The microscopic mechanism for the nitrogen depletion can be satisfactorily on the basis of a non-radiative exciton decay model.

\section{Experimental}

$\mathrm{N}$-rich copper nitride thin films (thickness $\sim 100 \mathrm{~nm}$ ) were deposited at room temperature (RT) by DC triode sputtering from a copper target in an $\mathrm{Ar}+\mathrm{N}_{2}$ atmosphere on glass and Si substrates. The elemental composition of the as-deposited samples was characterized by Rutherford backscattering spectroscopy (RBS) and non-RBS spectroscopy in combination with nuclear reaction analysis (NRA) techniques. A more detailed explanation about sample deposition and elemental characterisation procedures is described in Ref. [5].

Films were irradiated at RT with $\mathrm{Cu}$ ions at different energies from 10 to $42 \mathrm{MeV}$. The irradiations were performed in a high vacuum chamber (pressure $<10^{-4} \mathrm{~Pa}$ ) connected to the $5 \mathrm{MV}$ Tandem accelerator at the Helmholtz-Zentrum Dresden-Rossendorf. According to SRIM calculations [31] and assuming a copper nitride density of $5.89 \mathrm{~g} / \mathrm{cm}^{3}$ [32], the $S_{e}$ in the investigated energy range for this film thickness varies from 4.9 to $11.9 \mathrm{keV} / \mathrm{nm}$ over the whole film thickness and it is almost two orders of magnitude higher than the nuclear stopping power (i.e. $S_{\mathrm{n}}=0.07 \mathrm{keV} / \mathrm{nm}$ for $\mathrm{Cu}$ at $42 \mathrm{MeV}$ ). Thus, irradiations took place in an energy range clearly dominated by the electronic processes. The irradiation fluence was varied from $1 \times 10^{12}$ to $450 \times 10^{12} \mathrm{at} / \mathrm{cm}^{2}$. The beam current density was selected to prevent temperature enhancements higher than around $50^{\circ} \mathrm{C}$, which is clearly below to the reported values to observe significant nitrogen depletion [16]. Moreover, $\mathrm{X}$-ray diffraction data on similar samples heated up to $300^{\circ} \mathrm{C}$ [6] evidences no sample decomposition. Therefore, it can be concluded that the beam-induced temperature enhancement does not play a significant role in the described mechanism. Elastic recoil detection (ERD) technique was used to measure the $\mathrm{N}$ content during $\mathrm{Cu}$ irradiation. The recoiled nitrogen atoms were measured with a Bragg ionization chamber (for energies $>20 \mathrm{MeV}$ ) and with a ToF-E detector (for energies of 10 and $20 \mathrm{MeV}$ ). The ToF-E detector is located at a scattering angle of $44^{\circ}$. The analyzed area was about $2 \times 1 \mathrm{~mm}^{2}$ and it was characterized after the irradiations by measuring the size of the beam spot that was visible after irradiation. The charge was measured using the RBS signal from gold for a gold coated rotating vane that periodically intercepts the ion beam. This signal is calibrated against a Faraday cup. The fluence is calculated from both values. During the measurement intermediate spectrum files were saved to obtain the nitrogen contents as a function of fluence.

\section{Results}

The atomic concentration of nitrogen for as-deposited copper nitride thin films deposited both on glass and on Si substrates is determined by combining RBS, non-RBS and NRA measurements to be $33 \pm 2$ at.\% (overstoichiometric, N-rich layers). More details about the nitrogen content determination are reported in Ref. [5].

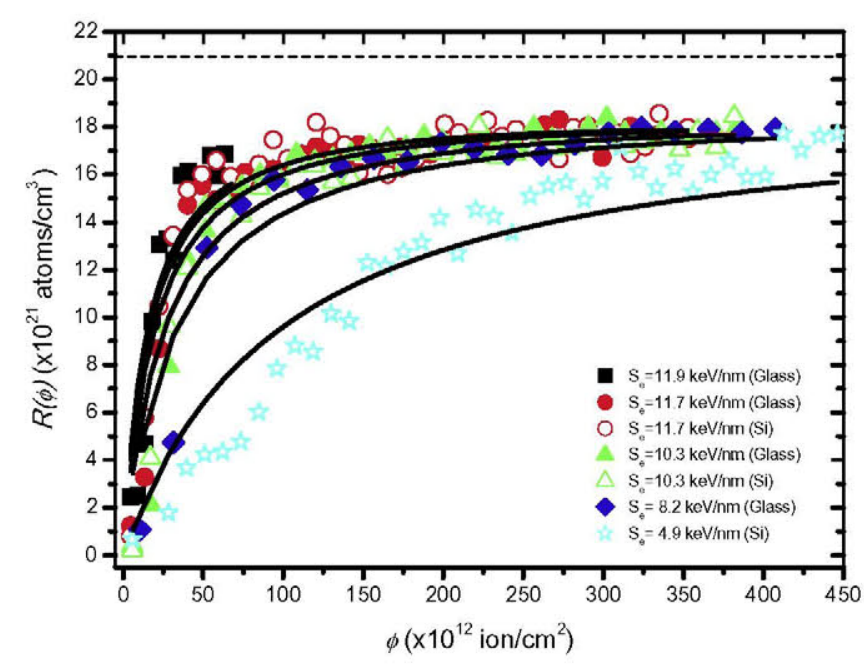

Fig. 1. Density of released nitrogen atoms for different $S_{e}(4.9-11.9 \mathrm{keV} / \mathrm{nm})$ as a function of the incoming ion fluence for $\mathrm{N}$-rich $\mathrm{Cu}_{3} \mathrm{~N}$ films deposited on both substrates $\mathrm{Si}$ and Glass. The black lines show the fits assuming the BRM and the parameters shown in Table 1 . The dotted line denotes the total density of nitrogen in the as grown samples.

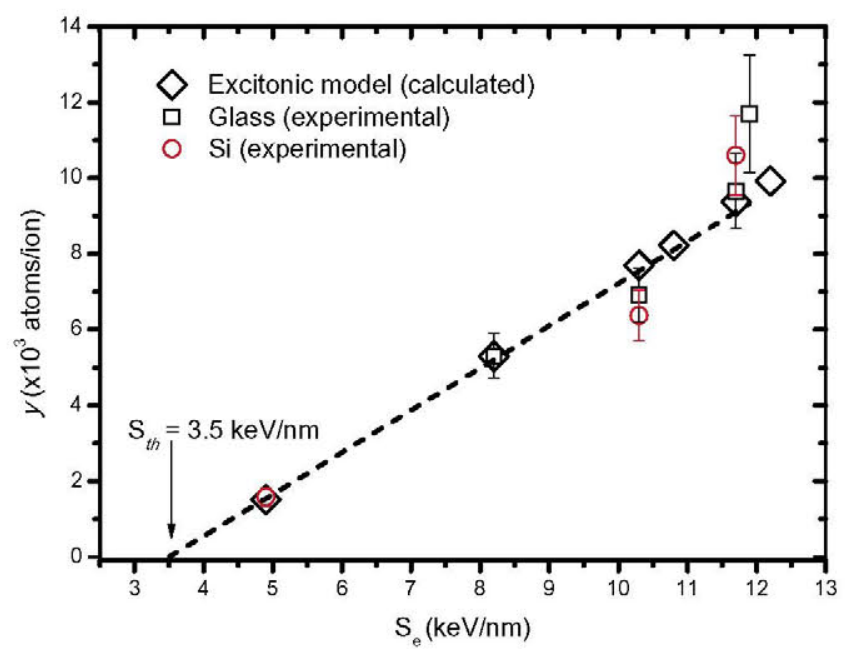

Fig. 2. Nitrogen sputtering yield $(y)$ as a function of ion stopping power for copper nitride films deposited on glass (open squares), Si (open circles). The diamonds correspond to the nitrogen sputtering yield calculated on the basis of the excitonic model. The dashed line denotes the linear fit and the extrapolation to zero yield to obtain the stopping power threshold value for nitrogen sputtering $\left(S_{t h}=3.5 \mathrm{keV} /\right.$ $\mathrm{nm}$ ). Note that the error bar for the points at $4.9 \mathrm{keV} / \mathrm{nm}$ is smaller than the marker size.

Previous swift heavy ion (SHI) irradiation experiments carried out in similar samples ascertained that, as in our case, $\mathrm{N}$ is sputtered away, whereas Cu remains in the films [14,15].

The overall yield $R(\phi)$ i.e. the density of sputtered $\mathrm{N}$ atoms per unit area for different $S_{e}\left(4.9>S_{e}>11.9 \mathrm{keV} / \mathrm{nm}\right)$ is depicted as a function of the total incoming ion fluence in Fig. 1 for $\mathrm{N}$-rich $\mathrm{Cu}_{3} \mathrm{~N}$ films deposited on glass $\left(\mathrm{Cu}_{3} \mathrm{~N} /\right.$ glass $)$ and on $\mathrm{Si}$ substrates $\left(\mathrm{Cu}_{3} \mathrm{~N} / \mathrm{Si}\right)$. The release of $\mathrm{N}$ for samples irradiated at the same fluence increases with rising $S_{e}$ for films grown on the two types of substrates (glass and $\mathrm{Si}$ ). Moreover, the $\mathrm{N}$ release curves present the same trend for both sets of samples. At low fluences, the nitrogen release per volume, $R(\phi)$, is approximately linear with fluence, and its rate $d R(\phi) / d \phi$, strongly decreases with increasing fluence. Finally, at high enough fluence, a saturation level in the nitrogen depletion is reached that corresponds to a low remaining nitrogen 
fraction $(5-10 \%)$. No nitrogen depletion is observed when further increasing the irradiation fluence. The saturation level is more rapidly reached for $S_{e}=4.9 \mathrm{keV} / \mathrm{nm}$ than for $S_{e}=11.7 \mathrm{keV} / \mathrm{nm}$.

The maximum unitary nitrogen sputtering yield, $y$ (i.e. per incident ion), is readily obtained from $d R(\phi) / d \phi$ at $\phi=0$, and it depends on electronic stopping power as shown in Fig. 2. It is observed that $y$ rises in a roughly linear way with increasing $S_{e}$. Extrapolation of the linear dependence in Fig. 2 down to the abscissa axis $(y=0)$ reveals that $\mathrm{N}$ release only takes place above a certain stopping power threshold $\left(S_{\text {th }}\right)$. By assuming that the linear dependence of $y$ on $S_{e}$ holds for $S_{e}<4.9 \mathrm{keV}, S_{t h}$ is estimated to be $\sim 3.5 \mathrm{keV} / \mathrm{nm}$.

\section{Discussion}

\subsection{Kinetic behavior}

The kinetic curves presented in this paper offer an interesting and novel set of data to describe the role of stopping power on the nitrogen sputtering yield in a metastable low bandgap semiconductor. The new results should provide relevant clues to explore the microscopic mechanisms that are responsible for SHI damage. As for other nitrides [28,30], oxides [33] or hydrides [29], nitrogen sputtering can be consistently explained on the basis of the BMR [29]. The equation describing the overall yield $R(\phi)$ as a function of fluence $\phi$, writes

$R(\phi)=\frac{\left(N_{0}-N_{f}\right)\left(1-e^{-k \phi}\right)}{1-\frac{N_{0}-N_{f}}{N_{0}} e^{-k \phi}}$,

where $N_{0}$ is the nitrogen density prior to irradiation, $N_{f}$ is the nitrogen density depleted from the film after irradiation, and $k$ is the molecular release cross section. According to the BMR model, the following steps have to operate in order to release nitrogen by swift heavy ion irradiation: (i) $\mathrm{Cu}-\mathrm{N}$ bonds are broken, thus, nitrogen atoms can be liberated from their lattice positions. (ii) Nitrogen atoms recombine in the bulk to form $\mathrm{N}_{2}$. This occurs whenever the bonds are broken within a short characteristic distance and within a time shorter than the lifetime of free nitrogen atoms in the damaged region (molecule formation faster than retrapping). (iii) Once a molecule is formed it diffuses out of the material without any further interaction.

Eq. (1) is used to fit the measurement data in Fig. 1 and the quality of the fits indicates that the BMR model is an adequate microscopic description for the nitrogen depletion process in this fluence range. As shown in Fig. 1, the curves tend to an asymptotic saturation level whose value is quite similar for all the samples, being in any case lower than the measured initial concentration of nitrogen, indicated in Fig. 1 by a dashed line. The fitting parameters are listed in Table 1, where the saturation level for the nitrogen depleted from the film, $N_{f}$, has been fixed at $\sim 18 \times 10^{21}$ atoms/ $\mathrm{cm}^{3}$. The normalized root-mean square deviation $(\sigma)$ to assess the goodness of the fits is also included in the Table 1 . The fact that films are not fully depleted of nitrogen even at very high

\section{Table 1}

Fitting parameters for the curves shown in Fig. 1. In this table, $S_{e}$ is the electronic stopping power, $k$ the molecular release cross section and $s$ the mean-square deviation. The nitrogen density depleted from the film after the irradiation $\left(N_{f}\right)$, is fixed at $18 \times 10^{21}$ atoms $/ \mathrm{cm}^{3}$.

\begin{tabular}{lllll}
\hline Substrate & Energy $(\mathrm{MeV})$ & $S_{e}(\mathrm{keV} / \mathrm{nm})$ & $k\left(\times 10^{-15} \mathrm{~cm}^{2}\right)$ & $\sigma$ \\
\hline Glass & 42 & 11.9 & $10 \pm 1$ & 0.82 \\
Glass & 40 & 11.7 & $7.6 \pm 0.1$ & 0.91 \\
Glass & 30 & 10.3 & $5.8 \pm 0.6$ & 0.90 \\
Glass & 20 & 8.2 & $4.5 \pm 0.5$ & 0.90 \\
Si & 40 & 11.7 & $9.0 \pm 0.1$ & 0.93 \\
Si & 30 & 10.3 & $5.7 \pm 0.6$ & 0.88 \\
Si & 10 & 4.9 & $1.5 \pm 0.2$ & 0.89 \\
\hline
\end{tabular}

irradiation fluences is explained in the BMR model by considering that the nitrogen density in the material at those high fluences is so low that molecule formation cannot occur (step (ii) cannot be fulfilled). From the experimental $N_{f}$ value the characteristic distance in a spherical volume to allow molecule formation is estimated to be $\ell_{c h}=\left(2 \cdot \frac{3}{4 \pi N_{f}}\right)^{1 / 3} \sim 0.3 \mathrm{~nm}$, not far from the value of $0.5 \mathrm{~nm}$ obtained by Adel et al. [29] for hydrogen pairing in irradiated a$\mathrm{C}: \mathrm{H}$. The calculated molecular release cross section linearly increases with $S_{e}$ in the measured range and has similar values for samples deposited on glass and on $\mathrm{Si}\left(k\right.$ around $5 \times 10^{-15} \mathrm{~cm}^{2}$, see Table 1$)$. The estimated values are smaller than those reported for InN $\left(k \sim 6.8 \times 10^{-12} \mathrm{~cm}^{2}\right)$ and GaN $\left(k \sim 0.6 \times 10^{-12} \mathrm{~cm}^{2}\right)$ $[28,34]$, possibly related to structural differences among materials.

\subsection{Microscopic physical mechanisms}

Nitrogen sputtering yields shown in Fig. 2 (up to $\sim 10^{4}$ atoms/ ion) are among the highest reported for sputtering of insulating, semiconductor and metallic targets, being comparable to those obtained for alkali halides, such as LiF [35]. These yields are orders of magnitude higher than those calculated for the nuclear collision regime ( $<0.16$ atom/ion) for $\mathrm{Cu}$ at $42 \mathrm{MeV}$, assuming a typical displacement energy of $25 \mathrm{eV}$ [32], which indicates that the mechanism for nitrogen depletion is of electronic nature.

Previous irradiation-induced sputtering data for $\mathrm{Cu}_{3} \mathrm{~N}$ irradiated with $\mathrm{Au}$ ion beam at $200 \mathrm{MeV}$ [36] have been qualitatively interpreted on the basis of the thermal spike model. Essentially the process can be described as vaporization or boiling of the damage track structure as a consequence of the thermal spike generated by the ion impact. This phenomenological model has been also used to describe high sputtering yields in several other materials (oxides, halides) when irradiated in the electronic regime. Nevertheless, a recent publication [6] has shown that the nitrogen sputtering yield in copper nitride films is independent of substrate temperature during irradiation within the $100-300^{\circ} \mathrm{C}$ range. This feature cannot be properly explained on the basis of the thermal spike model [19] since it would predict an enhancement of the damage track radius produced by each impact with temperature and thus, an increase in the overall sputtering yield. Therefore, we propose here to discuss the electronic sputtering process in terms of an alternative excitonic model [37,38], initially suggested by Itoh et al. [39], and satisfactorily applied to describe many damage features in $\mathrm{LiNbO}_{3}$ [37-42], as well as in alkali halides [43]. It assumes that the bond-breaking and nitrogen release from their lattice sites is caused by non-radiative decay of localized excitons. This is possible because the potential energy stored in every exciton (band-gap slightly higher than $1 \mathrm{eV}$ ) may be sufficient to break $\mathrm{N}-\mathrm{Cu}$ bonds. The sputtering yield $(y)$ per ion impact linearly depends on $S_{e}$ indicating that the energy needed to break a bond is always the same regardless of the temperature reached at the ion track. In other words, the bond-breaking efficiency is roughly proportional to the number of excitons generated by the ion impact and is independent of the temperature reached in the spike, provided that the temperature is high enough to promote nonradiative decay of excitons. The excitonic model $[37,38]$ assumes that the concentration profile of excitons around the ion trajectory (radial concentration) is coupled to the initial temperature profile and can be written as:

$N_{X}(r)=\frac{S_{e}}{\pi a_{0}^{2} I} \exp \left(-\frac{r^{2}}{a_{0}^{2}}\right)$,

where $S_{e}$ is the electronic stopping power, $I$ the energy required to form an electron-hole pair (typically 2-3 times the band-gap energy, i.e. $\sim 2 \mathrm{eV}$ for copper nitride), $r$ the radial distance, and $a_{0}$ the initial profile width. This parameter is related to the band-gap 


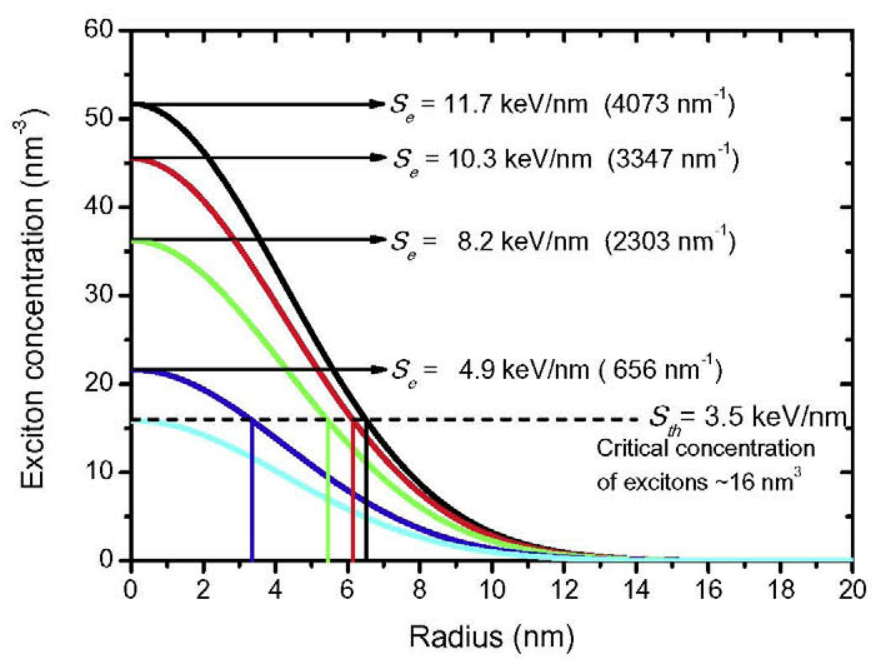

Fig. 3. Exciton concentration as a function of radius for different $S_{e}(4.9-11.7 \mathrm{keV} /$ $\mathrm{nm}$ ) used in this work and the profile corresponding to the threshold stopping power $\left(S_{t h}=3.5 \mathrm{keV} / \mathrm{nm}\right)$. Vertical lines indicate the distance at which the exciton concentration is equal to $n_{X c}$. The values in brackets correspond to the number of released nitrogen per nm around the ion track for every $S_{e}$.

energy by means of the electron-phonon mean free path [44], resulting in $a_{0} \sim 6 \mathrm{~nm}$ for copper nitride.

Fig. 3 shows exciton profiles corresponding to the ions used in this work (Table 1). In addition, the profile corresponding to the previously identified threshold stopping power $\left(S_{t h}=3.5 \mathrm{keV} / \mathrm{nm}\right)$ is plotted. The peak of the profile for $S_{e}=S_{\text {th }}$ provides the critical exciton concentration, $n_{X c}=16 \mathrm{~nm}^{-3}$, necessary for nitrogen release, i.e. $n_{X c}=2 /\left(4 / 3 \pi l_{c h}^{3}\right)$. This value corresponds to a characteristic distance, $l_{c h} \sim 0.3 \mathrm{~nm}$, which agrees quite well with that derived from the fitting of experimental data in Fig. 1 to the BMR model. This good agreement suggests that the non-radiative exciton decay may be the origin of the $\mathrm{N}-\mathrm{Cu}$ bond breaking.

If one integrates (cylindrical symmetry) the profiles obtained at every stopping power (Fig. 3) from the ion track centre $(r=0)$ up to the distance at which the exciton concentration equals $n_{X C}=16$ $\mathrm{nm}^{-3}$ (vertical lines in Fig. 3), one obtains the number of excitons per $\mathrm{nm}$ around the track (given in brackets in Fig. 3 ). This number is about two orders of magnitude larger than that of broken bonds, suggesting that the damage efficiency of the non-radiative exciton decay channel is quite low. This is not surprising since, luminescence measurements in alkali halides [44] indicate that only a small fraction $(\sim 1 \%)$ of the electron-hole pairs, generated during ionbeam irradiation, will contribute to the non-exciton decay.

The total number of sputtered nitrogen atoms calculated for copper nitride films on the basis of the non-radiative exciton decay approach by assuming that the efficiency is $\sim 2.5 \%$ is plotted in Fig. 3. A good agreement between calculated and experimental values is observed, which supports the description of electronic sputtering by means of the excitonic model. The fact that irradiation damage and nitrogen sputtering may be described on the basis of an exciton mechanism may also explain the decomposition of the copper nitride films when irradiating them with laser pulses and electrons where a significant thermal spike is not produced. Of course, a much better knowledge of the electronic properties of copper nitride and particularly of the exciton dynamics would be necessary before any sound model may be developed.

\section{Summary and conclusions}

Nitrogen sputtering yields under $\mathrm{Cu}$ irradiation have been measured in the energy range $10-42 \mathrm{MeV}$. Values are similar for films deposited on glass or silicon substrates and strongly increase with electronic stopping power up to around $10^{4} \mathrm{~N} /$ ion at $42 \mathrm{MeV}$ $(11.9 \mathrm{keV} / \mathrm{nm})$. The kinetics of sputtering can be described on the basis of the bulk molecular recombination model. A threshold for material decomposition has been estimated, by the first time, to be $\sim 3.5 \mathrm{keV} / \mathrm{nm}$. The high nitrogen sputtering yields and the threshold behavior indicate that sputtering mechanism are related to electronic excitation processes. Moreover, the consistency of the trends found for the measured nitrogen sputtering yields and those calculated on the basis the non-radiative exciton decay supports an excitonic model to be responsible for the experimental observations. The occurrence of a critical exciton density to promote a significant sputtering yield stands out the key role of the high electronic energy depositon rates achieved during swift heavy ion irradiation. Nevertheless, a better knowledge of the electronic properties of copper nitride films is necessary to further develop the excitonic model.

As a general conclusion, we expect that the reported data may provide new information on the electronic damage processes operating in low-bandgap semiconductors under SHI irradiation.

\section{Acknowledgements}

One of the authors (N.G.) acknowledge to the M.C.Y.T. Grant No. FIS 2008-01431 for financial support. Research by N. Gordillo was supported by a PICATA postdoctoral fellowship of the Moncloa Campus of International Excellence (UCM-UPM).

\section{References}

[1] G.H. Yue, P.X. Yan, J.Z. Liu, M.X. Wang, M. Li, X.M. Xuan, J. Appl. Phys. 98 (2005) 103506.

[2] N. Gordillo, R. González-Arrabal, A. Alvarez-Herrero, F. Agulló-López, J. Phys. D Appl. Phys. 42 (2009) 165101.

[3] C. Navio, M.J. Capitan, J. Alvarez, F. Yndurain, R. Miranda, Phys. Rev. B 76(2007) 085105.

[4] K. Venkata Subba Reddy, A. Sivasankar Reddy, P. Sreedhara Reddy, S. Uthanna, J. Mater. Sci.: Mater. Electron. 18 (2007) 1003.

[5] N. Gordillo, R. González-Arrabal, M.S. Martin-González, J. Olivares, A. Rivera, A Briones, F. Agulló-López, D.o. Boerma, J. Cryst. Growth 310 (2008) 4362.

[6] R. Gonzalez-Arrabal, N. Gordillo, M.S. Martin-González, R. Ruiz-Bustos, F. Agulló-López, J. Appl. Phys. 107 (2010).

[7] Z.Q. Liu, W.J. Wang, T.M. Wang, S. Chao, S.K. Zheng, Thin Solid Films 325 (1998) 55 .

[8] Z. Ji, Y. Zhang, Y. Yuan, C. Wang, Mater. Lett. 60 (2006) 3758.

[9] T. Nosaka, M. Yoshitake, A. Okamoto, S. Ogawa, Y. Nakayama, Appl. Surf. Sci. 169 (2001) 258.

[10] M. Asano, K. Umeda, A. Tasaki, Japan J. Appl. Phys. 29 (1990) 1985.

[11] L. Maya, Mater. Res. Soc. Symp. Proc. 282 (1993) 203.

[12] T. Mayurama, T. Morishita, Appl. Phys. Lett. 69 (1996) 890.

[13] R. Cremer, M. Witthault, D. Neuschütz, C. Trappe, M. Laurentis, O. Winkler, H. Kurz, Mikrochim. Acta 133 (2000) 299.

[14] S. Ghosh, F.Singh.D. Chaudhary, P. Shah, V. Ganesan, A. Gupta, D.K. Avasthi, Surf. Coat. Technol. 142 (2001) 1034.

[15] S. Gosh, V.V. Svakumar, A. Tripathi, S. Khan, V. Ganesan, A. Gupta, A. Nath, D.V. Avasthi, Nucl. Instrum. Meth. Phys. Res. B 248 (2006) 71.

[16] N. Gordillo, A. Rivera, R. Grötzschel, F. Munnik, D. Güttler, M.L. Crespillo, F. Agulló-López, R. González-Arrabal, J. Phys. D Appl. Phys. 43 (2010) 345301.

[17] R. Spohr, lon Tracks and Microtechnology, Basic Principles and Applications, Vieweg, Braunschweig 1990

[18] R.L. Fleischer, P.B. Price, R.M. Walker, Nuclear Tracks in Solids. Principles and Applications, University of California Press, Berkeley, 1975.

[19] F. Seitz, J.S. Koehler, Sol. Stat. Phys.:Adv. Res. Appl 2 (1956) 305.

[20] N. Itoh, Nucl. Instr. Meth. B 116 (1996) 33.

[21] N. Itoh, A. Marshall Stoneham, Nucl. Instr. Meth. B 146 (1998) 362.

[22] A. García-Navarro, F. Agulló-López, J. Olivares, J. Lamela, F. Jaque, J. Appl. Phys. 103 (2008) 093540.

[23] M. Toulemonde, W. Assmann, F. Grüner, C. Trautmann, Phys. Rev. Lett. 88 (2002) 057602.

[24] G. Szenes, Z.E. Horvath, B. Pécz, F. Pászti, L. Tóth, Phys. Rev. B 65 (2002) 045206.

[25] W. Wesch, A. Kamarou, E. Wendler, Nucl. Instr. Meth. Phys. Res. B 225 (2004) 111.

[26] A. Kamarou, W. Wesch, E. Wendler, A. Undisz, M. Rettenmayr, Phys. Rev. B 73 (2006) 184107

[27] C.S. Schnohr, P. Kluth, A.P. Byrne, G.J. Foran, M.C. Ridway, Phys. Rev. B 77 (2008) 073204. 
[28] S.K. Shrestha, H. Timmers, K. Scott, A. Butcher, M. Wintrebert-Fouquet, P. P-T Chen, Nucl. Instr. Meth. Phys. Res. B 234 (2005) 291.

[29] M.E. Adel, O. Amir, R. Cáliz, L.C. Feldman, J. Appl. Phys. 66 (1989) 3248.

[30] H. Timmers, T.D.M. Weijers, R.G. Elliman, J. Uribasterra, H.]. Whitlow, E.-L. Sarwe Nucl. Instr. Meth. Phys. Res. B 190 (2002) 428.

[31] J.F. Ziegler, M.D. Ziegler, J.P. Biersack, SRIM-2006.02

[32] Handbook of Chemistry and Physics, (84th ed.), CRC Press 2003.

[33] W.M. Arnoldbik, N. Tomozeiu, F.H.P.M. Habraken, Nucl. Instrum. Meth. B 190 (2002) 433.

[34] S.K. Shrestha, K. Scott, A. Butcher, M. Wintrebert-Fouquet, H. Timmers, Nucl. Instr. Meth. Phys. Res. B 219-220 (2004) 686.

[35] M. Toulemonde, W. Assmann, C. Trautmann, F. Grüner, H.D. Mieskes, H. Kucal, Z.G. Wang, Nucl. Instr. Meth. B 212 (2003) 346.

[36] S. Ghosh, A. Tripathi, T. Som, S.K. Srivastava, V. Ganesan, A. Gupta, D.K. Avasthi, Radiat. Eff. Defects Solids 154 (2) (2007) 151
37] F. Agulló-López, A. Méndez, G. García, J. Olivares, J.M. Cabrera, Phys. Rev. B 74 (2006) 174109

[38] A. Rivera, A. Méndez, G. García, J. Olivares, J.M. Cabrera, F. Agulló-López, J Lumin. 128 (2008) 703.

[39] N. Itoh, A.M. Stoneham, Materials Modifications by Electronic Excitation, Cambridge University Press, Cambridge, 2001.

[40] A. Rivera, J. Olivares, G. García, J.M. Cabrera, F. Agulló-Rueda, F. Agulló-López, Phys. Status Solidi A 206 (6) (2009) 1109

[41] A. Rivera, M.L. Crespillo, J. Olivares, G. García, F. Agulló-López, Nucl. Instr. Meth. B 268 (2010) 2249.

[42] A. Rivera, J. Olivares, G. Garcia, F. Agullo-Lopez, J. Phys.: Condens. Matter 24 (2012) 085401.

[43] L. Protin, Ph.D. Thesis, University of Caen (1994)

[44] M. Toulemonde, Ch. Dufour, A. Meftah, E. Paumier, Nucl. Instr. Meth. B $166-$ $167(2000) 903$. 